\title{
NEW METHODOLOGY FOR THE FARADAY ROTATION ANGLE RETRIEVAL IN THE SMOS FIELD OF VIEW
}

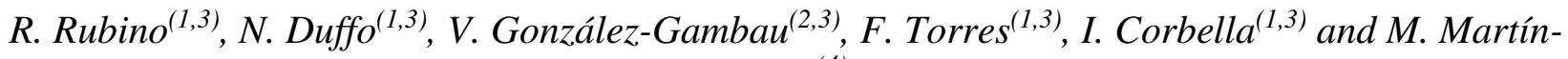 \\ Neira $^{(4)}$
}

\author{
(1)Remote Sensing Laboratory, Universitat Politècnica de Catalunya, Barcelona, Spain \\ (2)Institute of Marine Sciences, CSIC, Barcelona, Spain \\ (3)Barcelona Expert Centre, Barcelona, Spain \\ (4)European Space Agency, ESA-ESTEC, Noordwijk, The Netherlands
}

\begin{abstract}
In this work, a new methodology to estimate the Faraday Rotation Angle (FRA) from SMOS full-pol brightness temperature is presented. This approach is focused on retrieving the Vertical Total Electron Content (VTEC) and then, the FRA for most of the SMOS overpass, with the final aim of improving geophysical retrievals.
\end{abstract}

Index Terms- Faraday rotation, Vertical Electron Content, L-band radiometry, interferometry, Soil Moisture and Ocean Salinity (SMOS)

\section{INTRODUCTION}

After more than 8 years of operation, the ESA's SMOS (Soil Moisture and Ocean Salinity) instrument continues providing good quality Brightness Temperature (TB) data to generate frequent and global maps of soil moisture over the landmasses and surface salinity over the oceans. Its unique payload, MIRAS (Microwave Imaging Radiometer using Aperture Synthesis), is a two-dimensional synthetic aperture radiometer with multiple incidence angles and full polarimetric capabilities [1].

At MIRAS operating frequency, the Faraday Rotation (caused in the microwave radiation from the Earth when it propagates through the ionosphere) is not negligible. Therefore, it must be compensated. Currently, the Faraday Rotation Angle (FRA) is theoretically estimated by using a formulation that depends on external sources to provide ionospheric and geomagnetic field data. The magnitude of FRA can be estimated by using [2] [3]:

$$
\Omega_{f}=1.355 \times 10^{4} f^{-2} B_{0} \cos \Theta_{B} \sec \theta \mathrm{VTEC}
$$

with $f$ the frequency in $\mathrm{GHz}, \mathrm{B}_{0}$ the geomagnetic field at $400 \mathrm{~km}$ height in Tesla, $\Theta_{\mathrm{B}}$ the angle between the magnetic field and the wave propagation direction, $\theta$ the angle between the wave propagation direction and the vertical to the surface, and VTEC the vertical total electron content in TEC units $\left(10^{16}\right.$ electrons $\left./ \mathrm{m}^{2}\right)$.

In SMOS processing, the geomagnetic field is obtained from the database of the International Geomagnetic
Reference Field (IGRF) [4]. The ionospheric electron content VTEC is read from a SMOS auxiliary data file, i.e., the so-called "consolidated TEC," which gives the total electron content of the ionosphere for all the globe at intervals of $2 \mathrm{~h}$. For a given spatial direction from satellite to Earth, the longitude and latitude coordinates of the path crossing point through an altitude of $400 \mathrm{~km}$ are computed. Both the geomagnetic field and TEC maps are then interpolated at these coordinates, and also a time interpolation of the TEC map is applied (hereafter VTEC of the database).

Improved geophysical retrievals require getting the Faraday rotation directly from SMOS full-pol brightness temperature data in a continuous way. Latest advances in image reconstruction provide improved third and fourth Stokes parameters [5], which makes possible the FRA retrieval. This parameter can be estimated out from SMOS radiometric data by using the following equation (equivalent to equation (22) in [3]):

$$
\Omega_{f}=-\varphi-\frac{1}{2} \arctan \left(\frac{2 \Re\left(T_{B}^{x y}\right)}{T_{B}^{x x}-T_{B}^{y y}}\right)
$$

where $\varphi$ is the geometrical rotation angle, which is specific of the platform attitude and instrument orientation, and the terms $T_{B}^{x x}, T_{B}^{y y}, T_{B}^{x y}$ correspond to SMOS full-pol brightness temperature. Equation 2 can be used to estimate instantaneous FRA in the complete SMOS field of view. However, due to the large thermal noise, spatial bias and image reconstruction artifacts, FRA retrievals for a single snapshot present high errors.

A previous work showed that FRA could be directly retrieved at boresight from SMOS full-pol TB with good accuracy by using a smart spatio-temporal filtering strategy [6]. However, averaged boresight FRA estimations are not representative across the complete SMOS field of view (FoV), that is, if the averaged boresight FRA is assigned to all the pixels in the FoV, a large systematic bias appears across the FoV [7].

In this work, a new methodology for the FRA retrieval from SMOS radiometric data is proposed. It is based on deriving SMOS VTEC maps to use them in the FRA correction instead of the ones of the VTEC database by 
generating SMOS VTEC overpasses.

\section{FRA RETRIEVAL IN THE SMOS OVERPASS}

Every $2.4 \mathrm{~s}$, the radiometer onboard SMOS provides a block of full polarimetric L-band brightness temperature images in a large field of view with a swath of about $1000 \mathrm{~km}$. The FRA can be retrieved by introducing them in equation (2).

Due to MIRAS's relative poor radiometric sensitivity (thermal noise) and accuracy (spatial bias), FRA retrieval cannot be obtained in all the SMOS FoV with the required accuracy with a straightforward process. To mitigate these effects, both spatial and temporal averaging strategies are needed [6].

In our methodology, we first apply a temporal averaging on TB with a triangular filter every 15 snapshots. Once TB have been filtered, the FRA is retrieved by using equation 2 . The part of the SMOS field of view that is used in the processing corresponds to the zone where the sensitivity of TB to TEC is highest [8]. This zone is divided in three rectangles (top-left image in Figure 1).
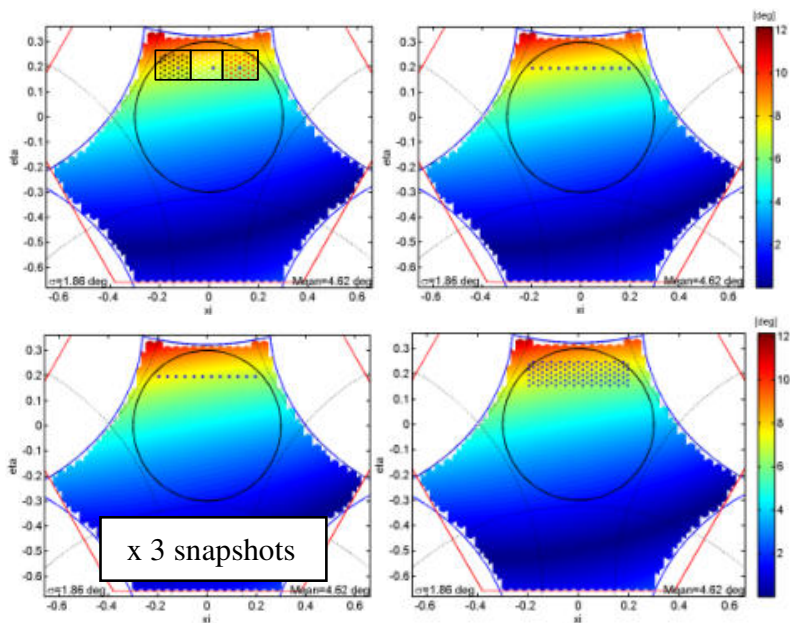

Figure 1. Scheme to illustrate the methodology for FRA retrieval. The image corresponds to a FRA snapshot theoretically estimated by using equation 1 and VTEC values from the database.

Then, the VTEC is calculated from the FRA values using equation 1. By doing the average in the three rectangles, 3 VTEC middle pixel values are obtained. In next step, an interpolation is done to obtain VTEC values in those pixels with the same eta, that is, in the 12 pixels located in the same line (see top-right image in Fig. 1). A weighted interpolation method has been used:

$$
V T E C_{i}=\frac{\sum_{j=1}^{3} \frac{V T E C_{j}}{d_{i j}^{2}}}{\sum_{j=1}^{3} \frac{1}{d_{i j}^{2}}}
$$

where subscript $j$ corresponds to the 3 pixels with averaged VTEC values, and subscript $i$ to the 12 pixels where VTEC is interpolated, and $d_{i j}$ is the distance between pixel $i$ and $j$.

The last step consists of doing a $2 \mathrm{D}$ interpolation with the weighted interpolation method in the longitude-latitude coordinates. We use a sliding window of 15 snapshots. The length of the window has been chosen to cover the part of the snapshot with the highest sensitivity of TB to TEC (see bottom-right image in Fig. 1). That is, 12 pixels of 15 consecutive snapshots are interpolated (see bottom-left image in Figure 1) to obtain all the pixels inside the three rectangles (i.e. 115 pixels) of the middle snapshot of the sliding window.

Following the proposed method, the VTEC in the most of the SMOS overpass is obtained. The final aim of this work is using this VTEC map derived from SMOS data to compensate the FR effects instead of using the VTEC of the database.

\section{FRA END-TO-END SIMULATOR}

In order to assess the performance of different FRA retrieval approaches, an end-to-end simulator has been developed in [7]. It makes use of a simple geophysical model of the Earth. Ground emissions are simulated depending on the target. For land, a constant brightness temperature of $280 \mathrm{~K}$ is used. For ocean, we use a Fresnel model considering a Sea Surface Salinity of 35 psu and a Sea Surface Temperature of $21^{\circ} \mathrm{C}$. To simulate SMOS polarimetric TB images at the antenna reference frame, we introduce the corresponding FRA value theoretically estimated from the auxiliary data. Once the synthetic brightness temperature images are available, simulated SMOS polarimetric visibility samples are computed by means of the G-matrix. Finally, a Moore-Penrose pseudoinverse is used to produce simulated SMOS full-pol polarimetric TB images. The simulator also takes into account the radiometric noise, which is included by adding the radiometric sensitivity by means of an uncorrelated random factor per pixel and per polarization with mean 0 .

In a first stage, the method proposed in section 2 was assessed in the simulator considering the effect of noise. Note that in this first analysis we are focused on the retrievals over ocean in the South Hemisphere. We use as reference the VTEC from the database to be compared to the VTEC retrieval from the simulator. Figure 2 shows both, the database VTEC (left), and the recovered VTEC from simulated data (right) for part (latitudes between $0^{\circ}$ to $-50^{\circ}$ ) of the overpass of SMOS over the Pacific in March $21^{\text {st }}$, 2011. Note that, as expected, the recovered VTEC has much better resolution than the database VTEC. The database VTEC is given in a coarse grid (a step of $2.5^{\circ}$ in latitude and $5^{\circ}$ in longitude) every 2 hours. Instead, our methodology recovers most of the SMOS overpass. 



Figure 2. VTEC of part of an orbit over the Pacific Ocean, March 21st, 2011. Left: Values from the database, right: VTEC values retrieved from simulated data with the proposed methodology.

The difference between the recovered VTEC and the database VTEC (used as reference) is shown in Fig. 3. Note the effect of discretization due to the lack of resolution of the database VTEC.

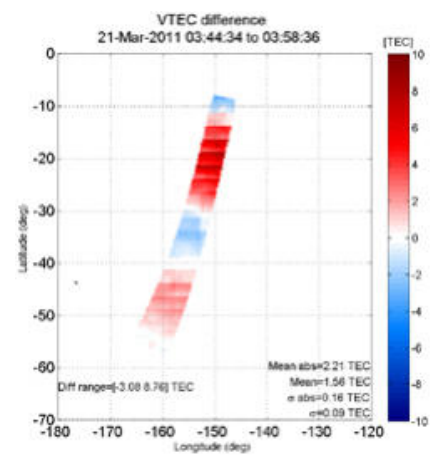

Figure 3. Difference between the retrieved VTEC and database VTEC (used as reference).

By using equation 1, the retrieved FRA can be obtained from the VTEC recovered from SMOS measurements for most of the SMOS FoV, which is the main purpose of this work. To analyze the performance of this simulated FRA retrieval methodology more in detail, Figure 4 shows the FRA recovery of the middle pixel of the rectangular zone (51 $1^{\circ}$ of incidence angle) vs latitude (green) and also the database FRA (red) in latitudes from $0^{\circ}$ to $-50^{\circ} \mathrm{S}$ of the orbit shown in the top of Fig. 1.



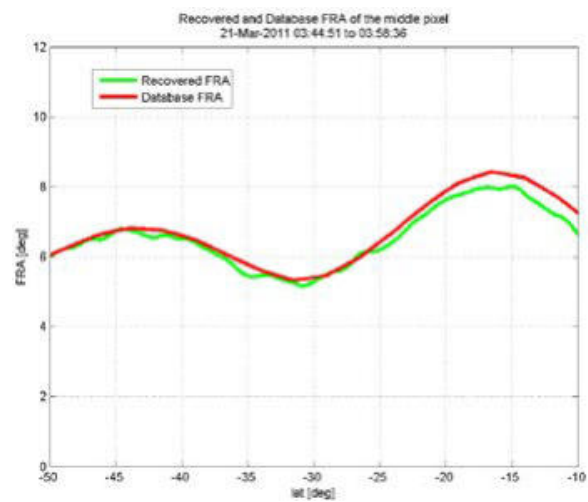

Figure 4. FRA of the middle pixel of the rectangle zone vs latitude: simulated recovered FRA in green and database FRA in red of the orbit shown in the top of the same figure.

\section{FRA RETRIEVALS FROM SMOS DATA}

On the basis of the promising results obtained with the theoretical images, we have analyzed the FRA retrievals from the SMOS TB data by applying the proposed methodology. Brightness temperature data have been processed by using MIRAS Testing Software (MTS), a tool developed at the Universitat Politècnica de Catalunya (UPC) that processes from the raw data up to brightness temperature images.

Fig 5 shows both, the database VTEC (left), and the recovered VTEC from the SMOS TB data (right).
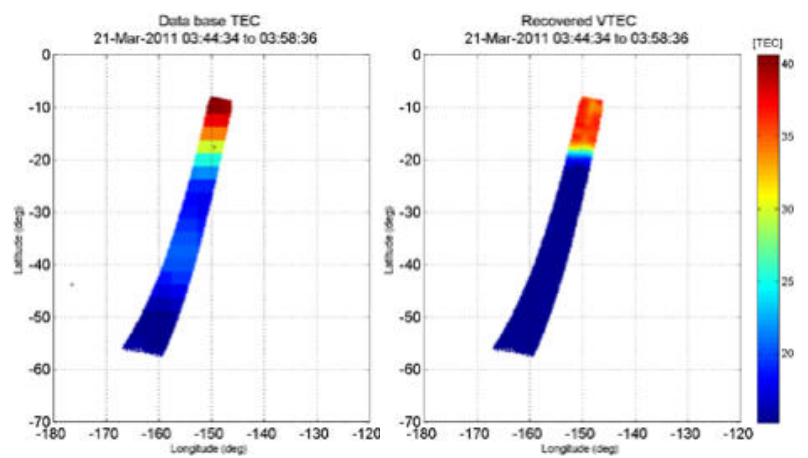

Figure 5. VTEC of an orbit over the Pacific Ocean, March 21st, 2011. Left: Values from the database, right: VTEC values retrieved from SMOS data with the proposed methodology.

The difference between the retrieved VTEC and the reference from the database is shown in Fig. 6 (left). These first results show a negative bias along the orbit, that is, the retrieved VTEC is generally below the database VTEC. The plot on the right shows the same magnitude once the mean value along the orbit has been subtracted. It can be appreciated that in the area where the database VTEC varies the most, it is where the differences are the highest. 

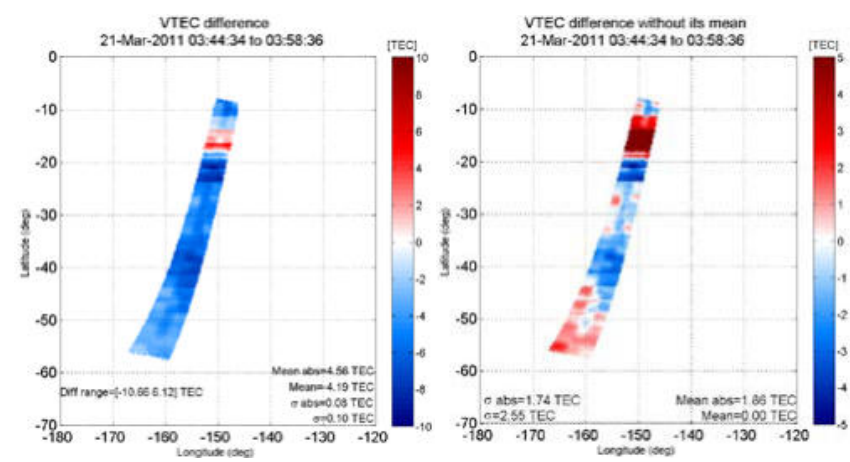

Figure 6. Left: Difference between the retrieved VTEC using SMOS data and the database VTEC. Right: The same magnitude once the mean value along the orbit has been subtracted.

The FRA recovery of the middle pixel of the rectangular zone from the SMOS TB data is plotted vs latitude in Fig 7. Also the database FRA values are shown for reference. A good agreement can be seen in the trace according to the fluctuation that it presents. However, it can be seen that there is a negative bias in the retrieved FRA with respect to the value of the database. This issue is currently under investigation.



Figure 7. FRA of the middle pixel of the rectangle zone vs latitude: recovered FRA from SMOS data in green and database FRA in red.

\section{CONCLUSIONS}

In this work, we propose a new methodology to retrieve the FRA from SMOS full-pol brightness temperature. This method is based on generating SMOS VTEC maps to compensate the effect of FR, instead of using the VTEC from the database. This would allow obtaining more accurate geophysical retrievals.

The simulator developed in [7] has been used to assess the performance of this new methodology. Simulations give promising results even though it was hard to compare it with a reference due to the fact that the existing databases have much worse resolution than the ones retrieved from SMOS recoveries.
First results with SMOS data show good performance with respect to the reference. However, we find a negative bias in the retrieval which is currently under investigation. We are currently working on the validation of these resulting SMOS VTEC maps by comparing them to combined GPS files [9].

Next step will be focused on correcting the TB by using the FRA retrieved from SMOS following the proposed methodology and evaluating the impact on the quality of the TB images.

\section{ACKNOWLEDGMENT}

This work has been supported by the European Space Agency and Deimos Engenharia (Portugal), SMOS P7 Subcontract DME CP12 no. 2015-005; ERDF (European Regional Development Fund) and by Spanish public funds, projects TEC2017-88850-R and ESP2015-67549-C3-1-R.

\section{REFERENCES}

[1] M. Martín-Neira et al., , "Polarimetric mode of MIRAS," IEEE Transactions on Geoscience and Remote Sensing, vol. 40, no. 8, pp. $1755-1768$, August 2002.

[2] D. M. Le Vine and S. Abraham, "The effect of the ionosphere on remote sensing of sea surface salinity from space: Absorption and emission at L band," IEEE Transactions on Geoscience and Remote Sensing, vol. 40, no. 4, pp. 771-782, April 2002.

[3] S. H. Yueh, "Estimates of Faraday rotation with passive Microwave polarimetry for microwave remote sensing of earth surfaces," IEEE Transactions on Geoscience and Remote Sensing, vol. 38, no. 5, pp. 2434-2438, September 2000.

[4] "International Geomagnetic Reference Field: The eleventh generation," Geophys. J. Int., vol. 183, no. 3, pp. 1216-1230, Dec. 2010.

[5] L. Wu et al. , "Radiometric performance of SMOS full polarimetric imaging," IEEE Geoscience and Remote Sensing Letters, vol. 10, no. 6, pp. 1454-1458, November 2013.

[6] I. Corbella et al., "Faraday Rotation Retrieval Using SMOS Radiometric Data". IEEE Geoscience \& Remote Sensing Letters, Vol.12, iss. 3, pp. 458-461. 2015.

[7] R. Rubino et al., "Direct faraday rotation angle retrieval in SMOS field of view," 2017 IEEE International Geoscience and Remote Sensing Symposium (IGARSS), pp. 697-698, 2017, doi: 10.1109/IGARSS.2017.8127047

[8] J.L. Vergely et al., "New total electron content retrieval improves SMOS sea surface salinity", J. Geophys. Res. Oceans, vol. 119, pp. 7295-7307, 2014, doi:10.1002/2014JC010150.

[9] Hernandez-Pajares, M. (2003), Performances of IGS ionosphere TEC maps, 7th IGS Iono WG report, UPC, Barcelona, Spain. 